\title{
THE ROLE OF TRADITION IN SYSTEMS ENGINEERING PRACTICE A PRELIMINARY ETHNOGRAPHIC STUDY
}

\author{
Paul D. Nugent, Western Connecticut State University, nugentp@wcsu.edu \\ Jennifer Flynn, Western Connecticut State University, flynnj@wcsu.edu
}

\begin{abstract}
This paper analyzes qualitative data from systems engineers at a large defense contracting company to consider the role of tradition in technical professional settings. We argue that among the various types of professions, tradition may play a critical and important role in the structuring and evaluation of complex technical work. While still preliminary, the analysis indicates that tradition protects core informal work processes from the encroachment of formal processes, and yet in other contexts tradition serves as starting point from which to conceive of rational organizational change. Potential contributions to theory and to practice are discussed.
\end{abstract}

Keywords: Professions, Organizational Change, Technology, Formal Process, Quality Control, Tradition, Trust

\section{INTRODUCTION}

Several decades ago the organizational researcher Stephen Barley argued persuasively that organizational theory and industrial research tend to make simplifying assumptions about the way in which work (especially technical work) is conducted and that very few actually analyze the work practices themselves (Barley, 1996; Barley \& Orr, 1997). While this has led to some hearing the call and exploring the intersection between the work itself and social structures and cultures, researchers continue to avoid studying how work is actually carried out and negotiated. This is unfortunate, as Barley and others provide empirical evidence for causal connections between work, technology, and organizational structure. For example, in a hospital the introduction of new medical technologies became an occasion for the doctors, nurses, and administrators to modify the work processes and structures (Barley, 1996). Ultimately, opportunities and constraints on agency are poorly explained by abstract theories, and instead are the result of workers consciously and collectively pursuing their task goals and interests.

Systems engineering is a form of professional work that may be fruitfully approached in this light. The primary author was a systems engineer for over 25 years at a large defense contracting company and has witnessed many types of change to the systems engineering work practices. In particular, the primary author has been immersed in this world in which informal norms regarding design processes and quality control were learned and internalized. These informal processes and bases of knowledge are passed on from generations of engineers to the next and, we believe, are best conceptualized as traditions. Tradition, therefore, can serve as a powerful framework for understanding certain types of organizational change from the point of view of the workers (engineers) themselves that is not carried out by formal processes and formal training. Let us first, however, consider how researchers have framed organizational change and how the concept of tradition may play a unique role.

\section{BACKGROUND AND LITERATURE REVIEW}

\section{Organizational Change}

Organizational change may occur based upon many factors and it is indeed virtually impossible to forge a theory or framework that will capture them in a parsimonious way (Poole \& Van de Ven, 2004). For example there are those who highlight planned versus unplanned change (Bennis, 1996) episodic versus continuous change (Weick \& Quinn, 1999), various levels of analysis, timeframes, and types of agency (Poole \& Van de Ven, 2004). One framework captures various "motors" of change including evolution in which variation, selection, and retention are key mechanisms, dialectic in which conflicts between parties lead to a new synthesis, life-cycle in which change it realized across sequences or stages, and teleology in which goal formation serves as a cybernetic guide for change (Van de Ven \& Poole, 1995).

Missing from these theories is a more explicit acknowledgement of the types of agency that we would expect in a professional bureaucracy, such as systems engineering, in which engineers collectively solve highly complex 


\section{Issues in Information Systems \\ Volume 20, Issue 1, pp. 224-232, 2019}

problems and design highly complex systems at a given site across decades. In these kinds of settings systems engineers develop informal ways of conducting work that we believe is best captured by the term tradition.

\section{Tradition}

We argue that the concept of tradition is central to understanding how professionals perform their work and conceive of organizational change. Concepts such as training, teaching, informal control, norms, values, culture, etc. partially capture practices and knowledge that are transmitted and reproduced across time, however they miss some important features that we believe are central to the concept of tradition and why it is important in work contexts such as systems engineering. Tradition is focused upon a relationship between the receivers and transmitters of knowledge/belief. According to one scholar of tradition:

Taken all together, this means that accepting and receiving tradition has the structure of belief. It is belief, since belief means accepting something as true and valid not on the basis of my own insight, but by relying on someone else. (Pieper, 2010, p. 18)

It is an essential part of the concept of tradition that no experience and no deductive reasoning can assimilate and surpass what is handed down. (Pieper, 2010, p. 19)

At one level, then, tradition provides a foundation for the establishment of beliefs and understandings so that those receiving the tradition are not starting from square one. In the context of work, this means that it is highly unlikely that a worker or engineer from the outside, no matter how experienced or intelligent, can simply be plugged into a situation and know what to do. The social, technical, and political histories that are being transmitted are all critical to understanding the work.

At another level, what differentiates tradition from other forms of knowledge transmission is that it implies a social relationship that exists across time in the minds of the actors. Those who have created the new knowledge/beliefs are aware that they are passing something on to newcomers. And those receiving the traditions do so with an acknowledgment that this knowledge is being passed to them consciously and with good intention. In tradition one acknowledges a sense of trust in the wisdom and authority of the past. Whether those from the past were viewed as heroes or simply as workers committed to a common project, those who came before created something that has the potential to be valuable today and needs to be transmitted and reproduced. This cultivates a sense of respect for those who created these understandings and wanting to become part of an ongoing legacy (Feldman, 1995). Beyond the objective tasks and production, tradition motivates because it represents an opportunity to be part of something bigger - participation in a historical process of building, innovating, and creating (Feldman, 1995).

Unfortunately, in broader society as well as in industry, tradition also implies a certain naiveté or stubborn unwillingness to consider potentially superior alternatives. Even in the social sciences there is a clear condescension to tradition as it is often framed as the opposite of the Enlightenment - a residue of the past that will eventually be burned off by pure rationality (Gerth \& Mills, 1946; Durkheim, 1957). And no one will argue that many traditions did need to be extinguished by rationality and science for moral or technical reasons, however at the same time scholars believe that without tradition we would also be without deeper meanings and sense of purpose (Shils, 1981).

It is no surprise, then, that some studies of tradition in organizations find that it is functional and not necessarily at odds with (or more appropriately it may be completely in line with) collective goals (Shils, 1981; Henriksen, 2001; Wiener et. al., 2001). More importantly, scholars of tradition are relaxing the preconception that traditions introduce inertia and resistance to change. Rather, as traditions are passed on there are opportunities to actively consider new alternatives. In other words, a clear theme is emerging in more recent scholarly framings of tradition that tradition also encompasses taken-for-granted and deeply rooted ways of interpreting the world and defining truth and is not necessarily resistant to change but rather is a reference point for being able to conceive of change (Bass, 2006; Camosy, 2012).

\section{Enterprise-Level Change Programs}

If we accept the notion that tradition can play a functional role in organizations, then it is also important to reflect on relatively recent movements within organizational fields that could counteract these benefits.

Over the last two or three decades virtually all professional bureaucracies have experienced the widespread adoption of what we will call enterprise-level quality control systems. For example in technical fields we see the emergence of Lean, Six Sigma, Capability Maturity Model Integration (CMMI), Lean, and Information Technology Infrastructure Library (ITIL). Similarly, in educational institutions and in the healthcare industry there are quality control initiatives that formally measure processes and strive for process optimization within a Quality Control (QC) philosophical 


\section{Issues in Information Systems \\ Volume 20, Issue 1, pp. 224-232, 2019}

paradigm. While these bureaucratic shifts can be seen as rational organizational responses to environmental pressures for legitimacy, their impact on internal effectiveness and efficiency remains somewhat in doubt (Shang \& Lin, 2009; Nugent \& Collar, 2014; Giroux \& Landry, 1998).

One could say this represents a tightening of Max Weber's "iron cage" of bureaucracy that may have negative social impacts on the professional workers. Indeed some studies have explored these effects and found forms of alienation and a shifting of responsibility/accountability from the professional to management and the "process owners." (Nugent, 2016). However, while bureaucratization takes away traditional or informal forms of control from the professional group, what, if any, are the countervailing forces that may resist or reverse this dynamic? In particular, as systems engineers represent a unique type of professional that we argue is more interdependent and social than other types of professionals, how do traditions influence the informal group? This is an important question because, we argue, in systems engineering settings a great deal of value-creation comes from and relies upon these traditions as opposed to impersonal bureaucratic structures borne from historical successes of QC and Total Quality Management (TQM) originally conceived for non-social technical systems.

\section{Systems Engineering and Tradition}

Much has been written about professional work and how it differs from other forms of work. For example, according to one of the fathers of sociology, Emile Durkheim, professions are characterized by a moral or ethical order that obliges its members to a particular duty or calling (Durkheim, 1957). In other words, over time these ethics and norms become internalized and can guide action through traditions rather than through formal means.

According to sociologists, in addition to this ethical character, professions can also be characterized by their jurisdictional claim to draw from an abstract body of knowledge to diagnose and treat various problems (Abbott, 1988). From this point of view, we can begin to consider what makes engineering in general, and systems engineering in particular, unique among the professions.

In professions such as medicine, law, and higher education, for example, the abstract body of knowledge used to diagnose and solve problems are relatively stable over time. Doctors need to keep up with advances in physiology and treatment, lawyers need to be aware of shifting and emergent laws, and professors need to keep pace with changes in student needs and in the world of research. In contrast, engineers design and build new and unique complex systems. Unlike the human body, a body of laws, or the body of academic/research concepts and theories, these systems possess highly unique sets of interfaces, internal dynamics, and configurations. The abstract body of knowledge representing a missile guidance system differs from that of the system that prepares and launches it in a myriad of ways. Unlike a doctor who can move from one human patient to the next with relative ease, the systems engineer working one system cannot easily or quickly be reassigned to another system because he or she must now learn an entirely new complex abstract body of knowledge.

Although this observation creates challenges (i.e. "ramp-up time") for a particular engineer moving from one system to the next, more importantly it evolves a social facet of systems engineering that is likely not as well-developed in the other professions. While doctors, lawyers, and professor may occasionally consult with one another or collaborate in some way, their day-to-day professional duties are carried out with less dependence on one another or the need to understand ever-changing technical, political, and social worlds. In contrast, systems engineering work is centered on developing system and subsystem requirements, architectural designs that will satisfy those requirements, test cases to ensure requirements have been met, and other various system-level tasks such as reliability, maintenance, security, information assurance, logistics, procedures, and supportability. Any one engineer at any one time performs these kinds of tasks and must interact with other engineers for information acquisition, information exchange, task coordination, and critical feedback.

Systems engineering then, as a profession, is highly social and organized in groups with differing functional and programmatic expertise/knowledge. It is also true that over time these groups develop their traditions, cultural frameworks, and ethics regarding their work and work quality. This forms the basis for what is often called traditional, clan, or informal control, and it is to this aspect of systems engineering and its sociological dynamics that we will now turn our attention.

\section{Informal versus Formal Control}

As many observers have noted, engineers and other technical professionals do form these close-knit groups and are governed as much by their home-grown codes regarding production processes and quality assessment as they are by any set of formal rules imposed upon them by the bureaucracy within which they are embedded (Adler, 2011; Kunda, 1992). 


\section{Issues in Information Systems \\ Volume 20, Issue 1, pp. 224-232, 2019}

Indeed, the sociologist Max Weber lamented that given its irrefutable efficiency the social form of organization known as bureaucracy would gradually erode or replace "traditional" forms of authority (Gerth \& Mills, 1946). Therefore, an interesting question that has preoccupied researchers throughout the last century is, to what extent has bureaucracy and its formal or "rational-legal" form of authority encroached upon the informal group leading to various forms of alienation? While much of this alienation was found to occur in non-professional occupations (i.e., wage laborers), the consensus, at least until the late $20^{\text {th }}$ century, was that professionals as a whole, with their ethical guidance and traditions, were granted more autonomy by management and therefore largely avoided these negative side effects (Becker, 1961; Blauner, 1964; Bonjean \& Grimes, 1970; Adler \& Borys, 1996).

However, as mentioned earlier, a new layer of bureaucracy embodying quality-control based enterprise-wide programs such as CMMI, Lean, Six Sigma, and ITIL has been implemented by professional institutions of all stripes in the last twenty to thirty years and poses potentially new threats to the traditions of the informal/traditional group because they often impose their own requirement for how work is to be performed. The few studies that have examined this bureaucratic shift have seen evidence of alienation along social dimensions as well as a shift in responsibility/accountability from the professional group to management and the "process owners" (Nugent, 2016). Given the widespread adoption of these kinds of enterprise-wide quality programs in technical fields as well as in education and healthcare, it is a bureaucratic shift that makes us ask what role tradition might play in these settings as professional workplaces are being forced to change.

To the extent that informal groups have evolved in these settings, these enterprise-wide bureaucratic structures represent an external source of change to which the group needs to adapt. Indeed, they represent a potential threat to these informal traditions as the bureaucratic system claims for itself certain jurisdictions such as quality assessment and the allocation of tasks/problems to specific individuals (Nugent \& Collar, 2014). Therefore, consistent with Weber's pessimism about the power of bureaucracy and of legal-rational authority, one can ask if and how countervailing forces may be at play. In other words, as informal professional groups engage in the work activities guided by their traditions, are there times when the formal structures are inadequate and they must create their own structure, processes, and therefore new traditions?

In this context, the purpose of this paper is to consider the role of tradition in confronting different types of organizational change. Ethnographic data drawn from two systems engineering groups in a large defense contracting company are analyzed in an attempt to explore these themes.

\section{METHOD}

The primary author was a systems engineer with over twenty five years of experience who collected ethnographic data (interviews and observations) from two different systems engineering groups at a large Navy defense contracting site in New England. The first (Group A) were systems engineers evolving the next generation of Trident II submarine embedded control system while the second (Group B) focused on incremental upgrades to a surface ship in a system-of-systems engineering context.

Group A represents a structural context in which the systems engineers experienced the implementation of an enterprise-level quality control process initiative (CMMI) and therefore provides data on how these engineers responded to threats to their traditional ways of performing their work and evaluating its quality. Therefore, this group was experiencing an externally imposed structural change.

Group B represents a structural context in which the systems engineers were experiencing growing pains with their change control process. Compared to Group A, the surface ship being developed is relatively new and therefore the focus was shifting from requirements and design to maintenance and the upgrading of components/systems onboard the platform. Therefore, this group was experiencing an internally imposed structural change.

Ethnography, as a research methodology, is evolving to address the challenges of outside observers interpreting social worlds. The manner in which subjects are framed and represented in ethnographic research has become a pivotal question in what some scholars refer to as the ontological turn in ethnography (Kelly, 2014). On the one hand, one of the ideals of science is to develop objective and universal theories to explain observable phenomena. Yet on the other hand the practice of science has been shown to be grounded in the social constructions - even in the "hard sciences" such as biology and physics (Latour, 1984; Knorr-Cetina \& Mulkay, 1983). 


\section{Issues in Information Systems \\ Volume 20, Issue 1, pp. 224-232, 2019}

While much of the debates in ethnography and anthropology revolve around the ontological and epistemological problem of an outsider gaining access to the meaning systems used by the insiders, the primary author of this study encountered the opposite problem. The primary author was, prior to the study, already a full-time systems engineer employed in various roles including requirements definition and system architecture design at the system, sub-system, and software component levels. Therefore, gaining access and developing rapport, or understanding the informal processes and traditions, were not central issues. The primary author already enjoyed "back-stage" access to and familiarity with social realities in meetings, customer reviews, peer reviews, and casual hallway conversations and was well aware of who the "key informants," or those most in tune with the organizational cultures, were.

Field-notes were recorded on an ad hoc basis and focused on contexts and discrete interactions in work activities and at meetings as well as capturing engineers' reactions to the introduction of new processes and technologies such as CMMI. These notes were initially hand-written in a journal but elaborated in computer word processing files at the end of the day. Over thirty interviews, lasting approximately one hour each, were recorded and transcribed over the research period.

Ethnographic interviewing methods were utilized in the data gathering phases (Lofland \& Loflan, 1984; Spradley, 1979). Consistent with these methods each interview began with "grand tour" questions such as "Can you tell me about when you came to the company and the different positions you have held?" and "Please describe what your current role, whom you work with, and the nature of your work interfaces and interactions." Probes were used to encourage the interviewee to elaborate on points or for clarification. Subsequent questions focused upon concrete work interactions that the interviewee recalled as being representative of good or poor working relations.

Grounded theory methods were used to evolve codes and to develop theory regarding the role of tradition in these two systems engineering structural situations (Strauss \& Corbin, 1990). The early coding phase was intentionally flexible and reflexive - allowing the data to stimulate thinking about theoretical categories and relationships while also allowing the emerging theory to guide the subsequent data gathering.

Early analysis of the data have been completed and preliminary results have been compiled.

\section{ANALYSIS}

Although the analysis is preliminary, relevant patterns are emerging. Tradition serves as both a constraint on change and as an existing structure from which to envision change. Each of these facets were clearly represented in the data but differently for each group.

\section{Functional Properties of Tradition}

At this site, systems engineers develop highly complex systems that requires extensive interaction with Department of Defense (DoD) customers, other defense contractors, and subject matter experts (SMEs) within the organization. Over time this has cultivated a strong sense of community across these groups and traditions centering on a commitment to the end users (Navy submariners) and to defect-free high quality "gold-plated" systems. More than a value system, however, this tradition permeates the way in which day-to-day tasks are performed with a sense of autonomy, empowerment, helpfulness, and a collective sense of what the end-product needs to be.

Therefore one of the more central traditions is an obligation for each systems engineer to accept responsibility for their product and this responsibility requires them to learn and understand the myriad of political, social, and technical expecations about the portion of the system they are working on.

In addition, each work group (program/function) develops its own work practices revolving around requirements elicitation, design tools and preferences, informal product reviews, and evaluations of quality. These traditions are home-grown and informal and only become visible in the interactions themselves and the expectations/obligations that they reveal.

\section{Tradition as a Legitimate Constraint on Change}

Group A generally resented the introduction of the new structure (CMMI) because it did not believe it led to internal performance improvements because these were fully and adequately achieved by existing formal processes, informal processes, and traditions. According to one systems engineer,

My own personal opinion of CMMI from a business point of view in a competitive environment, it is critical to have that as a shingle hanging on the door. It gives the appearance of a certain amount of predictability, it gives the appearance of manageability and control. To some extent you have manageability and control 
artifacts, but your product is not a set of artifacts. Your product is a system that works in the end. You know, if your system does not work but you have beautiful artifacts, it did no good whatsoever.

When asked if CMMI improved production another engineer stated,

No, not at all. It changed the artifacts that are produced, it changes individual accountability for producing artifacts. It drives up the costs in terms of the number of hours you have to expend - that cost has to be absorbed by the individual developers because costs can only go down - never up. The rationale is that this is going to make us cheaper. They really just put more pressure on individuals to follow more religion, produce more artifacts. So, what it comes down to is software is always developed the same way and you can tell that by watching a project. Projects typically start up, they get in some degree of trouble and how do they react when they get in trouble? And even senior management, even the evangelists and advocates of CMMI will start pulling people off of one project onto something else.

By formalizing some work processes and claiming responsibility for product quality Group A engineers also saw the implementation of CMMI as representing management's lack of trust in the veteran systems engineers. Furthermore, these engineers experienced a shift in responsibility and accountability away from the tradition and toward the formal bureaucracy. Therefore, an emerging theme is that the engineers "go through the motions" and put up with the "overhead" required for them to produce CMMI artifacts, but meanwhile strive to preserve the traditional informal processes that are ultimately responsible for producing a working product and evaluating its quality.

\section{Tradition as a Springboard for Change}

While Group A was reacting to and adapting to an externally imposed structural change, Group B was collectively recognizing a need for change. The existing change control process exhibited too many informal traditional elements leading to confusion and inefficiency. These process elements can be divided into those focused on rules and those focused on role assignments.

Change control in a defense contracting setting is a highly complex process. Systems engineers identify the need for changes/updates to hardware and software configuration items and enter detailed information into a change control application (IBM Rational Change). Those in administrator roles strive to train the engineers on the change control process, inspect new change proposals for compliance with process rules, and prepare agendas for change control meetings. Hardware and software changes are entered into the same system, but are addressed at different change control meetings with different technical leaders. The engineers proposing the changes attend these meetings and answer questions about the change that the technical lead may have. Decisions are made at these meetings regarding the progression of the change proposal across many status levels eventually leading to implementation. However along the way the proposals may be rejected or deferred in various ways.

All role occupants expressed frustration with the current state of this process. A great deal of the the process remained informal and grounded in traditions that had been evolving for years.

The existing traditions that were found to be insufficient were:

- Exception handling - lack of consistent rules

- Process differences for handling hardware versus software items - differences are arbitrary and dysfunctional

- Allocation of proposals to different change control boards - lack of consistent criteria

- What role occupants can initiate status transitions within the change control tool - handled informally on a case-by-case basis

- Rules for deferral - no consistent criteria

Therefore the group had evolved traditions in an organic manner that addressed contingencies in earlier generations of the system, however now as they were seeking efficiency and consistency these traditions were no longer adequate. In addition, role responsibilities and inter-group communications were often defined informally including:

- Who is responsible for the change control board meeting agenda and action items

- Who is responsible for screening items for process compliance prior to the meetings

- Lack of feedback to engineers when items were rejected due to process non-compliance 
Therefor from a role/responsibility point of view, these systems engineers understood that the existing work traditions were inadequate and new more formalized processes needed to be defined.

Critically it is only from the point of view of existing traditions that the new processes and new traditions could be conceived. That is, the new processes were not being conceived in a vacuum but rather within a context of existing traditions and informal processes that could no longer adequately address the complexity of the change control processes for known reasons. That is, these traditions were modified and therefore not replaced.

In this context the engineers did not resent the change or view it as a lack of trust on the part of management. Rather, even though it disadvantaged some while advantaging others in terms of work obligations, there was a clear consensus that it was a rational and necessary collective goal. Systems engineers actively consider the political, social, and technological implications of change and do so in a conscious and deliberate manner that modifies past traditions to accommodate new goals and to overcome perceived problems.

\section{SUMMARY AND RECOMMENDATIONS}

\section{Implications for Theory}

Scholars of tradition have acknowledged its dual aspect in which, on the one hand, it can resist change, and on the other it can serve as a necessary vantage point from which to conceive of new structures and forms. And from our analysis it is clear that both of these dynamics occur and both of them are dependent upon actors understanding their core values and understanding the legitimacy of new goals in order for change to branch down the correct paths. In other words, in neither case is tradition an unconscious or naïve resistance to change.

As Barley argued quite a long time ago, when one looks at the way in which work is actually performed and change actually enacted, one is able to cultivate new theories and explanations that simplifying theories tend to miss. The concept of tradition in a systems engineering work context helps us to more fully appreciate how change occurs over time. With Group A we saw that the systems engineers protected their traditional ways of designing and evaluating work because they believed it was much more effective to achieve goals than the externally imposed quality control programs such as CMMI. In contrast, Group B saw that the existing traditions were problematic and needed to be modified, yet they also served as a necessary reference point from which to conceive of the new processes. Without the concept of tradition, as an informal means to pass along best-practices and knowledge across time, there would be no convenient theoretical way to explain what happened in both Groups A and B. Tradition bridges notions of both permanence and change from the point of view of goal-oriented collective action (agency) (Sewell, 1992).

From an organization theory perspective, this reinforces some of the basic theories of the New Institutionalism that identify a functional role for a buffering between a group's internal (informal/traditional) structure and the formal (Powell \& DiMaggio, 1991). Indeed, management's micro-managing of the work group can lead to peceptions of distrust and violate ceremonial norms:

Considerations of face characterize ceremonial management. Confidence in structural elements is maintained through three practices - avoidance, discretion, and overlooking. Avoidance and discretion are encouraged by decoupling autonomous subunits; overlooking anomalies is also quite common. Both internal participants and external constituents cooperate in these practices.

...Evaluation and inspection are public assertions of societal control which violate the assumption that everyone is acting with competence and in good faith. Violating this assumption lowers morale and confidence. Thus, evaluation and inspection undermine the ceremonial aspects of organizations. (Powell \& DiMaggio, 1991, pp. 58, 59)

In particular, this buffering function requires that the superiors/managers trust the internal groups and refrain from evaluating their outputs.

\section{Implications for Practice}

Systems engineering managers need to be aware that over years and decades their engineers come to understand the work products, processes, customer preferences, social relationsthips, technologies, and quality control at a deep level and evolve their own informal traditions that lie at the very heart of value creation in systems engineering contexts.

The imposition of formal processes onto their work introduces the potential for conflict and perceptions of lack of trust because it often implies that management and "process owners" understand the work at the same level. In 


\section{Issues in Information Systems}

Volume 20, Issue 1, pp. 224-232, 2019

addition it represents a shift in accountability and responsibility away from the informal traditions to the formal bureaucracy that can weaken the value-creating potentials of processes rooted in tradition. While in some industry settings this may indeed lead to an overall improvement in performance, it is unlikely that this will be the case in systems engineering because of the idiosyncracies of technical, political, and social worlds the engineer needs to understand and maintain.

On the other hand, the informal group is also willing and ready to shift away from existing informal traditions when they collectively sense the need to do so. Rather than imposing change from above, management is better advised to clarify goals so that the systems engineers can effect change from within as they are the ones that are most intimate with the details of the processes.

Ultimately, however, the main contribution of our paper is to argue that tradition is an important concept that enables us to more fully appreciate how work groups manage work-related knoweldge across time. Especially in a systems engineerting context, the work flow would be entirely crippled if these traditions were ignored or extinguished. Enterprise-level change programs tend to ignore this role of tradition and need to be implemented in manner that acknowledges and respects the value creation that lies at the heart of these traditions.

\section{REFERENCES}

Abbott, A. (1988). The system of professions: an essay on the division of expert labor. Chicago: Univ. of Chicago Press.

Adler, P. (2011). The sociological ambivalence of bureaucracy: from Weber via Gouldner to Marx. Organization Science online. March 23, 244-266.

Adler, P. \& Borys, B. (1996). Two types of bureaucracy: enabling and coercive. in Administrative Science Quarterly, 41, 61-89.

Barley, S. R. (1996). Technicians in the workplace: ethnographic evidence for bringing work into organization studies. Administrative Science Quarterly, 41, 404-441.

Barley, S. R. \& Orr, J. E. (eds.) (1997). Between craft and science: technical work in U.S. settings. ILR Press: Ithaca.

Bass, R., (2006). The role of tradition in concealing and grounding truth: two opposing freudian legacies on truth and tradition. American Imago, 63(3), 331-353.

Becker, H. (1961). Boys in white. Chicago: University of Chicago Press.

Bennis, W. G. (1966). Changing organizations. New York: McGraw-Hill.

Blauner, R. (1964). Alienation and freedom. Chicago: University of Chicago Press.

Bonjean, C. M. \& Grimes, M. D. (1970). Bureaucracy and alienation: a dimensional approach. Social Forces, 48(3), 365-373.

Camosy, C. C. (2012). The role of normative traditions in bioethics. The American Journal of Bioethics, 12(12).

Durkheim, E. (1957). Professional ethics and civic morals. Routledge.

Feldman, S. P. (2007). Moral memory: why and how moral companies manage tradition. Journal of Business Ethics, 72, 395-409.

Gerth, H. \& Mills, W. (1946). From max weber: essays in sociology. Oxford University Press. 
Giroux, H. \& Landry, S. (1998). Schools of thought in and against total quality. Journal of Managerial Issues, $10(2)$.

Henriksen, L. (2001). Knowledge management and the missing tradition - on the role of tradition, knowledge and competencies in industrial production. Studies in Cultures, Organisations, and Societies, 7, 81-104.

Kelly, J. D. (2014). Introduction: the ontological turn in French philosophical anthropology. Journal of Ethnographic Theory, 4(1).

Knorr-Cetina, K. D. \& Mulkay, M. (1983). Science observed: perspectives on the social study of science. London: Sage.

Kunda, G. (1992). Engineering culture. Temple University Press: Philadelphia.

Latour, B. (1984). War and peace of microbes. The pasteurization of France 1-150. Cambridge, MA: Harvard University Press.

Lofland, J. \& Lofland, L. H. (1984). Analyzing social settings. Wadsworth Inc.

Nugent, P. D. (2016). Bureaucracy 2.0: the bureaucratization of professional work. Issues in Information Systems Journal, 17(I), 128-135.

Nugent, P. D. \& Collar, E. (2014). The hidden perils of addressing complexity with formal process: a philosophical and empirical analysis. Complex Systems Design and Management.

Pieper, J. (2010). Tradition: concept and claim. St. Augustine's Press.

Poole, M. S. \& Van de Ven, A. H. (Eds.) (1996). Handbook of organizational change and innovation. Oxford.

Powell, W. W. \& DiMaggio, P. J. (Eds.) (1991). The new institutionalism in organizational analysis. The University of Chicago Press, 1991.

Sewell W. H. (1992). A Theory of structure: duality, agency, and transformation. American Journal of Sociology, 98(1), 1-29.

Shang, S. \& Lin, S. (2009). Understanding the effectiveness of Capability Maturity Model Integration by examining the knowledge management of software development processes. Business and Economics Management, 20(5).

Shils, E. (1981). Tradition. University of Chicago Press.

Spradley, J. P. (1979). The Ethnographic Interview. Fort Worth: Harcourt Brace Javonovich College Publishers.

Strauss, A. \& Corbin, J. (1990). Basics of qualitative research: grounded theory procedures and techniques. Sage Publications.

Van de Ven, A. H. \& Poole, M. S. (1995). Explaining development and change in organizations. Academy of Management Review, 20, 510-540.

Weick K. \& Quinn, R. E. (1999). Organizational change and development. Annual Review of Psychology, 50, 361386.

Wiener, M., Remus, U, Heumann, J., \& Mahring, M. (2015). The effective promotion of informal control in information systems offshoring projects. European Journal of Information Systems, 24, 569-58 